\title{
Clinical implications of age and sex in the prevalence of periodontitis in Korean adults with diabetes
}

\author{
KYUNGDO HAN $^{1}$ and JUN-BEOM PARK ${ }^{2}$ \\ Departments of ${ }^{1}$ Biostatistics and ${ }^{2}$ Periodontics, College of Medicine, \\ The Catholic University of Korea, Seoul 06591, Republic of Korea
}

Received October 4, 2017; Accepted December 21, 2017

DOI: $10.3892 /$ etm.2018.5880

\begin{abstract}
The present study was performed to assess the risk factors for periodontitis in Korean adults with diabetes. Data from the Korean National Health and Nutrition Examination Survey of the Korean population, conducted between January 2012 and December 2014 were used in the investigation. The presence of periodontitis in participants with diabetes in association with demographic variables and the anthropometric characteristics of the participants was investigated. Multiple logistic regression analyses were used to assess the associations between periodontitis and age, sex, diabetic control and duration of diabetes, following adjustment for confounding factors. The odds ratio of periodontitis was higher in individuals $\geq 65$ years old compared with individuals $<65$ years old (1.152). The odds ratio of periodontitis was significantly higher in males compared with females (1.774). The number of patients with moderate and severe periodontitis differed significantly between different age groups. The present study revealed that age, sex and oral health behavior are risk indicators for periodontitis in patients with diabetes. The present study suggests that that an increased age, being male and engaging in poor oral health behavior increases the risk of periodontitis in participants with diabetes.
\end{abstract}

\section{Introduction}

Diabetes mellitus is a complex metabolic disorder. A key feature of diabetes mellitus is the reduced function of the $\beta$ cells in the islets of Langerhans in the pancreas. These $\beta$ cells produce insulin and their reduced function may decrease insulin secretion and lead to increased blood glucose levels (1). The synthesis of advanced glycosylation end-products and the induction of oxidative stress may exacerbate early pathological vascular changes (2). In patients with diabetes, associated

Correspondence to: Dr Jun-Beom Park, Department of Periodontics, College of Medicine, The Catholic University of Korea, 222 Banpo-daero, Seoul 06591, Republic of Korea

E-mail: jbassoonis@yahoo.co.kr

Key words: age, diabetes mellitus, periodontitis, risk factors, sex complications may contribute to increased morbidity and premature mortality (3). Retinopathy, neuropathy, cardiovascular disease, nephropathy and encephalopathy are considered to be the primary complications of diabetes (4) and periodontal disease has been implicated as the sixth complication of diabetes (5). Periodontitis is a common, chronic, inflammatory disease that induces the gradual destruction of the supporting apparatus of the teeth, causing teeth to become mobile, which may ultimately result in tooth loss (6). It has been reported that the prevalence of periodontal disease in diabetic patients is $>85 \%$ (27.3\% of patients had gingivitis and $59.5 \%$ had periodontitis) whereas the prevalence of periodontitis in the general population is $46 \%(1,7)$. Diabetes is a known risk factor of periodontal disease and patients with diabetes have an increased prevalence of severe periodontitis compared with healthy adults (8). Indeed, it has been demonstrated that the susceptibility to periodontitis increases by $\sim 3$-fold in patients with diabetes (6). Among individuals with chronic periodontitis, patients with diabetes exhibit enhanced lipopolysaccharide-induced immune responsiveness, implicating an exacerbated inflammatory response (9).

It has been suggested that diabetes and periodontitis are biologically linked (10) Hyperglycemia associated with diabetes has been previously studied due to its association with adverse periodontal outcomes (3). However, to the best of our knowledge, the risk factors for periodontitis specifically in patients with diabetes have not yet been identified. Therefore, the present study was performed to assess the risk factors for periodontitis in Korean adults with diabetes using nationally representative data.

\section{Materials and methods}

Survey and subjects. The results of the present study were obtained by secondary analysis of data obtained during the Korean National Health and Nutrition Examination Survey (KNHANES) between January 2012 and December 2014. The KNHANES was approved by the Institutional Review Board of the Korea Center for Disease Control and all participants provided written informed consent. The Institutional Review Board at the Catholic University of Korea approved the present study (KC14EISI0636).

A total of 23,626 individuals participated in the KNHANES survey. The number of individuals included in the present 
study was reduced to 1,630 following the exclusion of participants $<30$ years old, individuals that had only undergone $<8 \mathrm{~h}$ fasting prior to examination, participants with missing values, individuals without diabetes mellitus and participants that did not undergo an oral examination (Fig. 1).

Demographic variables. The sociodemographic and lifestyle variables of the participants were evaluated by a self-administered questionnaire that assessed education level, household income, smoking, alcohol intake and physical exercise. Regarding smoking status, patients were classified as non-smokers, ex-smokers or current smokers. Non-smokers were those who had never smoked or had smoked $<100$ cigarettes during their lifetime, whereas ex-smokers were those who had smoked $>100$ cigarettes in their entire life but were not smoking at the time of the study. Regarding alcohol consumption, patients were categorized as non-drinkers, mild to moderate drinkers (1-30 g/day) or heavy drinkers ( $>30 \mathrm{~g} /$ day). Physical activity was measured using the international physical activity questionnaire as part of the KNHANES. Subjects who exercised for $\geq 5$ occasions per week for 30 min per session, or those who participated in strenuous physical activity for $\geq 3$ occasions per week for $20 \mathrm{~min}$ per session, were defined as regular exercisers. Education level was categorized as either high school education ( $\geq 10$ years schooling) or middle school graduate or lower ( $<10$ years schooling). The number of household members was evaluated. Household income was divided by the number of included family members and categorized into quartiles. The lowest quartile of household income was $<$ USD 1,092.40 per month and patients were categorized as being in the lowest quartile or not. The participant's self-reported health status was categorized as good or not good (which consisted of patients reporting their health as average or bad).

Anthropometric measurements. Anthropometric measurements of individuals that participated in the present study were taken by the trained staff members. Participants wore light, indoor clothing without shoes for the measurement of body weight and height, as previously described (11). The narrowest point between the iliac crest and the lower border of the rib cage was used to determine waist circumference. The following formula of weight $/ \mathrm{height}^{2}\left(\mathrm{~kg} / \mathrm{m}^{2}\right)$ was used to calculate body mass index. A standard mercury sphygmomanometer (Baumanometer; W.A. Baum Co., Inc., Copiague, NY, USA) was used to measure systolic and diastolic blood pressure in the right arm. Blood pressure measurements were performed twice with a 5 min interval and the average of the two measurements was used for analysis. Blood samples were collected from the antecubital vein of each participant to measure the white blood cell count. The white blood cell count was measured using an automated hematology analyzer (XE-2100D; Sysmex Corporation, Kobe, Japan).

Definition of diabetes mellitus and hypertension. A high waist circumference was categorized as $\geq 90 \mathrm{~cm}$ in men and $\geq 80 \mathrm{~cm}$ in women. Hypertension was diagnosed if an individual had a systolic blood pressure of $>160 \mathrm{~mm} \mathrm{Hg}$ or a diastolic blood pressure of $>90 \mathrm{~mm} \mathrm{Hg}$, or if the individual was using systemic antihypertensive drugs. A fasting blood cholesterol level $>240 \mathrm{mg} / \mathrm{ml}$ or the use of medication for the condition was considered to indicate the presence of hypercholesterolemia. The level of kidney function was determined by estimating the glomerular filtration rate (eGFR) using the following equations: eGFR $\left(\mathrm{ml} / \mathrm{min} / 1.73 \mathrm{~m}^{2}\right)=186.3 \mathrm{x}$ (serum creatinine $\left.{ }^{-1.154}\right) \mathrm{x}\left(\mathrm{age}^{-0.203}\right.$ ) for males and 186.3x (serum creatinine $\left.^{-1.154}\right) \times\left(\right.$ age $\left.^{-0.203}\right) \times 0.742$ for females. Serum creatinine was measured by the colorimetric method using a Hitachi 7600 modular chemistry analyzer (Hitachi, Ltd., Tokyo, Japan). The eGFR was categorized as either $\geq 60$ or not as previously described (12). Cardiovascular disease was considered present if the individual had experienced a stroke or had a congenital heart defect (13).

Diabetes was defined as a fasting blood sugar $>126 \mathrm{mg} / \mathrm{dl}$ or the individual was currently using anti-diabetic medication (14). The recognition, treatment and control of diabetes was self-evaluated, as well as insulin injection. Glucose and glycated hemoglobin levels were categorized into five levels. Patients were classified into the following 5 groups based on their glycated hemoglobin levels: $<6,6 \leq x<6.5,6.5 \leq x<7$, $7 \leq x<7.9$ and $\geq 8$ and into the following 5 groups based on their glucose levels: $<100,100 \leq x<120,120 \leq x<140,140 \leq x<160$ and $>160$.

Periodontitis and oral health behaviors. The oral health status of the participants was evaluated using the World Health Organization Community Periodontal Index (CPI) $(15,16)$. The index teeth according to the Federation Dentaire Internationale system were 11, 16, 17, 26, 27, 31, 36, 37, 46 and 47 (17). Periodontitis was defined as CPIs of 3 and 4 . The CPI score was 3 in cases of a shallow pocket with a depth of 3.5-5.5 $\mathrm{mm}$ and 4 in cases of a deep pocket with a depth $\geq 5.5 \mathrm{~mm}$ (18). Moderate periodontitis was defined as a CPI of 3 and severe periodontitis was defined as a CPI of 4 .

The frequency of daily tooth brushing and the use of secondary oral products were used to evaluate oral health behavior. Dental floss, interdental brushes, electric toothbrushes, irrigation devices, end-tufted brushes, tongue cleaners, mouthwash and special devices for dentures were considered to be secondary oral products. The survey also recorded the participant's self-reported oral status, the presence of any toothache, chewing discomfort, speech discomfort and whether patients had undergone a dental checkup within that year.

Statistical analysis. The results are presented as the mean \pm standard error of the mean for continuous variables and as proportions (standard errors) for categorical variables. To assess differences in characteristics as categorized by body mass index, a $\chi^{2}$ test was performed to assess categorical variables and an independent $t$ test was used to assess continuous variables. The risk factors for periodontitis in diabetes were evaluated following adjustments for age, sex, smoking, drinking, number of household members, income, self-reported health, medication, self-reported oral status, tooth pain, frequency of tooth brushing per day and the use of secondary oral products. Multiple logistic regression analyses were used to assess the associations between periodontitis and age, sex, diabetic control and duration of diabetes, following adjustment for confounding factors. $\mathrm{P}<0.05$ was considered to indicate a statistically significant difference. All statistical 


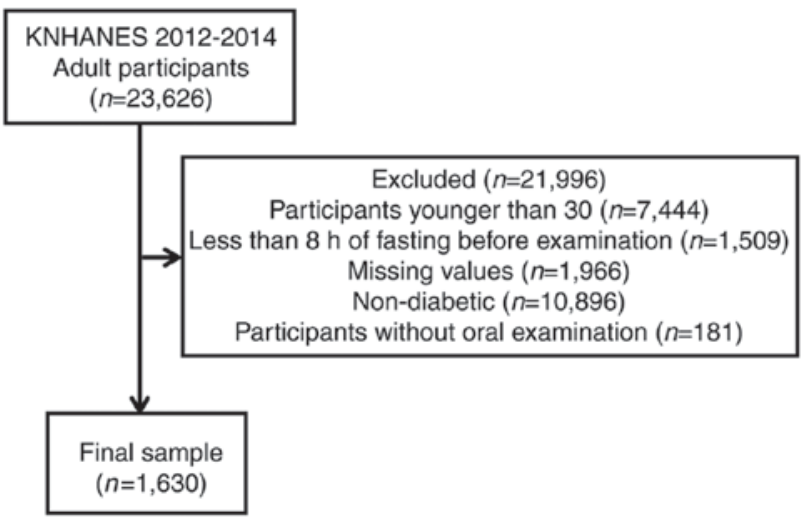

Figure 1. Flow chart of participants included and excluded from the study. KNHANES, Korean National Health and Nutrition Examination Survey.

analyses were performed using SAS software version 9.2 for Windows (SAS Institute, Inc., Cary, NC, USA).

\section{Results}

Baseline characteristics. Table I details the baseline characteristics of the participants included in the study according to the presence of periodontitis. The odds ratio (OR) of periodontitis was higher in individuals aged $\geq 65$ years old compared with individuals aged 30-65 years old (OR 1.152). The OR of periodontitis was significantly higher in males compared with females, with a $95 \%$ confidence interval of 1.387-2.269 $(\mathrm{P}<0.05)$. Being male, smoking, drinking, have a higher number of household members, being in the lowest income quartile, have an average or bad health status, having problematic self-reported oral status, experiencing tooth pain, experiencing chewing or speech discomfort, having a lower frequency of tooth brushing and not using secondary oral products were significantly associated with the incidence of periodontitis in the diabetic population.

The odds ratio of periodontitis following adjustments for confounding factors. ORs and their $95 \%$ confidence intervals for moderate and severe periodontitis categorized by various variables following adjustments for age, sex, smoking, drinking, number of household members, income, self-reported health, medication, self-reported oral status, tooth pain, frequency of tooth brushing per day and use of secondary oral products are presented in Fig. 2. The ORs and 95\% confidence intervals of periodontitis were $1.60(1.05,2.46)$ and $2.28(1.47,3.52)$ for ex-smokers and current smokers, respectively when non-smokers were considered as a reference. The ORs and 95\% confidence intervals of periodontitis for the individuals were $1.51(1.01,2.26)$ and $1.20(0.91,1.58)$ for the individuals with a toothbrushing frequency of $\leq 1$ and 2 , respectively when participants with toothbrushing frequency of 3 was considered as a reference. These results indicate that individuals with current smoking had higher ORs of periodontitis. Similarly, participants with a toothbrushing frequency of $\leq 1$ had higher ORs of periodontitis.

The percentage of moderate and severe periodontitis categorized by different variables. Fig. 3 presents the percentage of moderate and severe periodontitis categorized by age, sex, duration of diabetes and glycated hemoglobin. The percentages of moderate periodontitis in individuals aged $30-39$ and $\geq 70$ were 24.1 and $36.8 \%$, respectively. The percentages of moderate periodontitis in male and female were 35.5 and $30.2 \%$, respectively. The percentage of patients with periodontitis differed significantly between different age groups $(\mathrm{P}=0.0074)$ and significant differences were also noted between males and females $(\mathrm{P}<0.0001)$. There were no significant differences between patients with moderate and severe periodontitis.

\section{Discussion}

The present study used nationally representative data to determine that age, sex and oral health behavior were risk factors for periodontitis in Korean adults with diabetes. The effect of age on the prevalence of periodontitis may partially be explained by the onset of chronic inflammation, which is a common feature of aging and age-associated diseases, including periodontitis (19). It has been previously revealed that the prevalence and severity of periodontal disease increased as age increased (1). Similarly, periodontitis occurred at a higher rate in diabetic participants compared with non-diabetic individuals and this phenomenon was positively correlated with age in individuals with diabetes (19). It has been suggested that patients with diabetes aged $\geq 35$ years old experience more rapid destruction of the periodontium (20). Furthermore, it has been demonstrated that age is significantly associated with the increased prevalence and greater severity of destructive periodontal disease in patients with diabetes (21). The results of present study confirmed the importance of age in determining the prevalence of periodontitis in patients with diabetes.

Differences between the two sexes have also been noted in previous studies. A previous report revealed that diabetic males had worse periodontal conditions compared with diabetic females (22). A previous study also revealed statistically significant associations between males and severe periodontitis (23). These results therefore suggest that there is a greater need for the regular periodontal evaluation and effective oral hygiene care among males with diabetes than in females with diabetes to decrease the risk of developing periodontitis and progression of periodontitis into a more severe form (24). The present study revealed that the percentage of patients with moderate and severe periodontitis did not significantly differ between different duration of diabetes or diabetic control. In a previous study, diabetic status was associated with an increased severity and prevalence of destructive periodontal disease in the diabetic population (21). Furthermore, glycemic status influences the prevalence and severity of periodontal disease in diabetic participants (1). Individuals with poorly controlled diabetes and periodontitis are more likely to suffer from gingival bleeding than those with good or moderate control (25). Conversely, no significant correlation was observed between periodontitis and glycated hemoglobin levels in non-diabetic subjects (26). Similarly, glycemic control, defined by fasting plasma glucose $(140 \mathrm{mg} / \mathrm{dl})$ and glycohemoglobin value $(6.5 \%)$, was not significantly associated with periodontal status (27). Diabetic control did not reveal any differences in the periodontal condition (20). A previous report demonstrated 
Table I. Baseline characteristics of study participants with moderate and severe periodontitis.

\begin{tabular}{|c|c|c|c|c|}
\hline Characteristic & $\begin{array}{l}\text { No periodontitis, } \\
\mathrm{n}=838, \% \text { (SEM) }\end{array}$ & $\begin{array}{c}\text { Periodontitis, } \mathrm{n}=792 \\
\%(\mathrm{SEM})\end{array}$ & P-value & $\begin{array}{l}\text { Odds ratio } \\
(95 \% \mathrm{CI})\end{array}$ \\
\hline Age (years) & & & 0.4059 & \\
\hline $30-64$ & $67.4(1.8)$ & $65.3(2.1)$ & & 1 (reference) \\
\hline$\geq 65$ & $32.6(1.8)$ & $34.7(2.1)$ & & $1.152(0.916,1.449)$ \\
\hline Sex & & & $<0.0001^{\mathrm{a}}$ & \\
\hline Male & $49.3(2.1)$ & $60.9(2)$ & & $1.774(1.387,2.269)$ \\
\hline Female & $50.7(2.1)$ & $39.1(2)$ & & 1 (reference) \\
\hline Smoking & & & $<0.0001^{\mathrm{a}}$ & \\
\hline No & $60.4(2.2)$ & $43.5(2)$ & & 1 (reference) \\
\hline Ex & $21(1.8)$ & $24.9(1.8)$ & & $1.514(0.993,2.31)$ \\
\hline Current & $18.6(1.6)$ & $31.6(2.1)$ & & $2.503(1.67,3.752)$ \\
\hline Drinking & & & $0.0362^{\mathrm{a}}$ & \\
\hline Non-drinker & $37.3(1.9)$ & $30.8(2)$ & & 1 (reference) \\
\hline Mild to moderate drinker & $54(2.1)$ & $57.1(2.1)$ & & $1.176(0.9,1.535)$ \\
\hline Heavy drinker & $8.7(1.3)$ & $12.1(1.4)$ & & $1.388(0.855,2.255)$ \\
\hline Exercise & & & 0.3669 & \\
\hline No & $83.9(1.8)$ & $86.1(1.8)$ & & 1 (reference) \\
\hline Yes & $16.1(1.8)$ & $13.9(1.8)$ & & $0.832(0.559,1.237)$ \\
\hline Number of household members & & & $0.0079^{\mathrm{a}}$ & \\
\hline 1 & $10.5(1.2)$ & $13.6(1.5)$ & & 1 (reference) \\
\hline 2 & $30.9(1.8)$ & $36.7(2.2)$ & & $0.793(0.544,1.156)$ \\
\hline$\geq 3$ & $58.6(2)$ & $49.7(2.5)$ & & $0.626(0.414,0.949)$ \\
\hline Education & & & 0.2500 & \\
\hline Middle-school graduate or lower & $51.2(2.2)$ & $54.8(2.2)$ & & 1 (reference) \\
\hline High-school education or higher & $48.8(2.2)$ & $45.2(2.2)$ & & $0.924(0.689,1.24)$ \\
\hline Income (the lowest quartile) & & & $0.0157^{\mathrm{a}}$ & \\
\hline No & $76.1(1.7)$ & $69.8(2.2)$ & & 1 (reference) \\
\hline Yes & $23.9(1.7)$ & $30.2(2.2)$ & & $1.312(0.979,1.757)$ \\
\hline Self reported health status & & & $0.0122^{\mathrm{a}}$ & \\
\hline Good & $17.4(1.6)$ & $12.3(1.3)$ & & 1 (reference) \\
\hline Average or bad & $82.6(1.6)$ & $87.7(1.3)$ & & $1.67(1.19,2.343)$ \\
\hline Body mass index $\left(\mathrm{kg} / \mathrm{m}^{2}\right)$ & & & 0.2601 & \\
\hline$<25$ & $47.8(2.1)$ & $51.2(2.1)$ & & 1 (reference) \\
\hline$\geq 25$ & $52.2(2.1)$ & $48.8(2.1)$ & & $0.939(0.735,1.199)$ \\
\hline $\begin{array}{l}\text { High waist circumference, } \geq 90 \mathrm{~cm} \\
\text { in men and } \geq 80 \mathrm{~cm} \text { in women }\end{array}$ & & & $0.0793^{\mathrm{a}}$ & \\
\hline No & $44(2.1)$ & $49(2.1)$ & & $1.091(0.852,1.396)$ \\
\hline Yes & $56(2.1)$ & $51(2.1)$ & & 1 (reference) \\
\hline Hypertension & & & 0.1316 & \\
\hline No & $47(2.1)$ & $42.4(2.2)$ & & 1 (reference) \\
\hline Yes & $53(2.1)$ & $57.6(2.2)$ & & $1.052(0.809,1.367)$ \\
\hline Hypercholesterolemia & & & 0.8652 & \\
\hline No & $69.2(1.9)$ & $69.7(1.9)$ & & 1 (reference) \\
\hline Yes & $30.8(1.9)$ & $30.3(1.9)$ & & $1.04(0.79,1.37)$ \\
\hline Estimated glomerular filtration rate $\left(\mathrm{ml} / \mathrm{min} / 1.73 \mathrm{~m}^{2}\right)$ & & & 0.7636 & \\
\hline$\geq 60$ & $91.7(1.1)$ & $92.1(1)$ & & $1.197(0.796,1.801)$ \\
\hline$<60$ & $8.3(1.1)$ & $7.9(1)$ & & 1 (reference) \\
\hline Cardiovascular disease & & & 0.9456 & \\
\hline No & $90.9(1.1)$ & $90.8(1.1)$ & & 1 (reference) \\
\hline Yes & $9.1(1.1)$ & $9.2(1.1)$ & & $0.912(0.615,1.352)$ \\
\hline Diabetes (recognition) & & & 0.3699 & \\
\hline No & $37.6(2.2)$ & $40.2(2.2)$ & & 1 (reference) \\
\hline Yes & $62.4(2.2)$ & $59.8(2.2)$ & & $0.785(0.611,1.008)$ \\
\hline
\end{tabular}


Table I. Continued.

\begin{tabular}{|c|c|c|c|c|}
\hline Characteristic & $\begin{array}{l}\text { No periodontitis, } \\
\mathrm{n}=838, \% \text { (SEM) }\end{array}$ & $\begin{array}{c}\text { Periodontitis, } \mathrm{n}=792, \\
\%(\mathrm{SEM})\end{array}$ & P-value & $\begin{array}{l}\text { Odds ratio } \\
(95 \% \mathrm{CI})\end{array}$ \\
\hline Diabetes (treated) & & & 0.1644 & \\
\hline No & $43.4(2.2)$ & $47.7(2.2)$ & & 1 (reference) \\
\hline Yes & $56.6(2.2)$ & $52.3(2.2)$ & & $0.732(0.567,0.944)$ \\
\hline Diabetes (controlled) & & & 0.9387 & \\
\hline No & $78.2(1.9)$ & $78(1.9)$ & & 1 (reference) \\
\hline Yes & $21.8(1.9)$ & $22(1.9)$ & & $0.993(0.728,1.353)$ \\
\hline Insulin injection & & & 0.4330 & \\
\hline No & $94.8(0.8)$ & $93.8(1)$ & & 1 (reference) \\
\hline Yes & $5.2(0.8)$ & $6.2(1)$ & & $1.150(0.663,1.994)$ \\
\hline Diabetic medication & & & 0.234 & \\
\hline No & $45(2.2)$ & $48.6(2.2)$ & & 1 (reference) \\
\hline Yes & $55(2.2)$ & $51.4(2.2)$ & & $0.758(0.59,0.973)$ \\
\hline Glucose level & & & 0.9500 & \\
\hline$<100$ & $9.8(1.1)$ & $9.4(1.2)$ & & 1 (reference) \\
\hline $100 \leq x<120$ & $22.5(1.7)$ & $22.2(1.9)$ & & $1.095(0.725,1.655)$ \\
\hline $120 \leq x<140$ & $33.9(2.1)$ & $32.6(2.1)$ & & $1.079(0.721,1.615)$ \\
\hline $140 \leq x<160$ & $15.6(1.6)$ & $15.9(1.4)$ & & $1.146(0.724,1.812)$ \\
\hline$\geq 160$ & $18.2(1.7)$ & $19.9(1.7)$ & & $1.326(0.852,2.065)$ \\
\hline Glycated hemoglobin & & & 0.4800 & \\
\hline$<6$ & $5.8(1.1)$ & $7.8(1.1)$ & & 1 (reference) \\
\hline $6 \leq x<6.5$ & $16(1.6)$ & $14.2(1.6)$ & & $0.701(0.4,1.231)$ \\
\hline $6.5 \leq x<7$ & $27.6(1.9)$ & $28.6(2.1)$ & & $0.805(0.468,1.386)$ \\
\hline $7 \leq x<7.9$ & $31.1(1.9)$ & $28.1(1.9)$ & & $0.699(0.418,1.168)$ \\
\hline$\geq 8$ & $19.5(1.7)$ & $21.3(1.7)$ & & $0.91(0.526,1.577)$ \\
\hline Dental checkup within a year & & & 0.2490 & \\
\hline No & $76.7(1.7)$ & $73.8(1.9)$ & & 1 (reference) \\
\hline Yes & $23.3(1.7)$ & $26.2(1.9)$ & & $1.184(0.887,1.579)$ \\
\hline Self-reported oral status & & & $<0.0001^{\mathrm{a}}$ & \\
\hline Favorable & $14.9(1.6)$ & $9.9(1.2)$ & & 1 (reference) \\
\hline Average & $36.6(2.1)$ & $26.5(1.7)$ & & $1.179(0.772,1.801)$ \\
\hline Problematic & $48.6(2.2)$ & $63.6(1.9)$ & & $2.019(1.346,3.027)$ \\
\hline Tooth pain & & & $<0.0001^{\mathrm{a}}$ & \\
\hline No & $65.4(2)$ & $47.6(2.1)$ & & 1 (reference) \\
\hline Yes & $34.6(2)$ & $52.4(2.1)$ & & $2.086(1.653,2.632)$ \\
\hline Chewing discomfort & & & $<0.0001^{\mathrm{a}}$ & \\
\hline No & $69.7(2)$ & $56.9(2.1)$ & & 1 (reference) \\
\hline Yes & $30.3(2)$ & $43.1(2.1)$ & & $1.642(1.271,2.12)$ \\
\hline Speech discomfort & & & $0.0339^{\mathrm{a}}$ & \\
\hline No & $85.5(1.4)$ & $81.1(1.6)$ & & 1 (reference) \\
\hline Yes & $14.5(1.4)$ & $18.9(1.6)$ & & $1.277(0.946,1.724)$ \\
\hline Frequency of tooth brushing per day & & & $0.0019^{\mathrm{a}}$ & \\
\hline$\leq 1$ & $12.5(1.4)$ & $18.5(1.7)$ & & 1 (reference) \\
\hline 2 & $42.6(2.2)$ & $46.1(2)$ & & $0.773(0.534,1.119)$ \\
\hline$\geq 3$ & $44.9(2.2)$ & $35.4(2)$ & & $0.583(0.393,0.865)$ \\
\hline Use of secondary oral products & & & $0.0020^{\mathrm{a}}$ & \\
\hline No & $54.1(2.2)$ & $63.6(2.1)$ & & 1 (reference) \\
\hline Yes & $45.9(2.2)$ & $36.4(2.1)$ & & $0.757(0.583,0.981)$ \\
\hline
\end{tabular}

Data are presented as percentages (standard error of the mean) of the study participants. ${ }^{a} \mathrm{P}<0.05$. CI, confidence interval. SEM, standard error of the mean. 


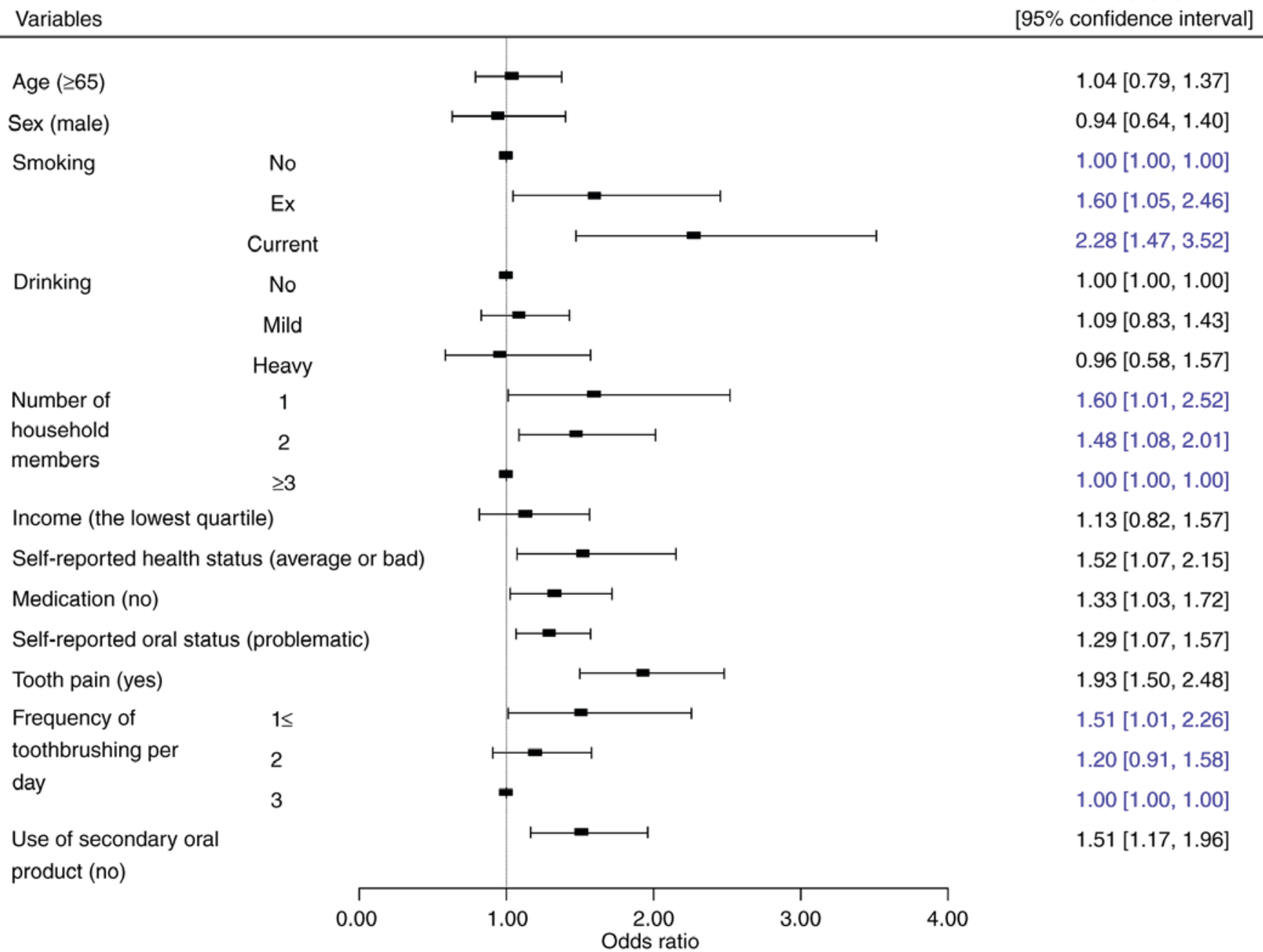

Figure 2. The odds ratio and their $95 \%$ confidence intervals for moderate and severe periodontitis categorized by different variables. Blue text indicates values that were significant.

that the glycated hemoglobin levels were not significantly correlated with periodontal values in diabetic participants (28), which was similar to trends observed in the present study. The association between the duration of diabetes and periodontitis was evaluated in the present study; however no significant association was observed. The association between the duration of diabetes and periodontitis is controversial as some reports have demonstrated that the two factors were associated, while others have reported no association $(19,20,25,27,28)$. A previous study reported that the number of years since the diagnosis of diabetes was more significant than age for predicting the severity of periodontal disease in diabetic patients (29). A previous study observed that the duration of diabetes mellitus had an association with the prevalence and severity of periodontal disease (1). It should be noted that the number of participants in these previous reports were relatively small, being composed of only 100 patients (46 males and 54 females) (29) and 1,500 patients (751 males and 749 females), respectively (1). However, no correlation was observed between periodontitis in individuals with diabetes and the length of time from the diagnosis of diabetes (19). Several previous studies have reported that the duration of diabetes was not associated with the periodontal condition $(20,27,28)$. A previous report revealed that no significant correlation was observed between diabetic duration and gingival bleeding (25). An advantage of the present study is that it was based on a nationally representative sample of Korean patients with diabetes, which allows for an effective investigation into whether age and sex are associated with periodontitis. The sampling units were based on the population and housing census conducted by the National Census Registry in Korea and survey sample weights were adjusted for participation and response rates (30). To the best of our knowledge, the present study is the first to demonstrate the importance of age and sex as risk indicators for the prevalence of periodontitis in individuals with diabetes (31). The present study assessed the effects of various risk indicators by subgroup analysis, including age, sex, control of diabetes and duration of diabetes. However, the present study did have certain limitations. Firstly, as it was a cross-sectional study it was not possible to identify a cause-and-effect association. Secondly, the present study used partial-mouth recording protocols of CPI, which may underestimate the prevalence of periodontal disease. To overcome these limitations a prospective study using full-mouth recording may be designed, however this type of study requires far greater resources to complete. 


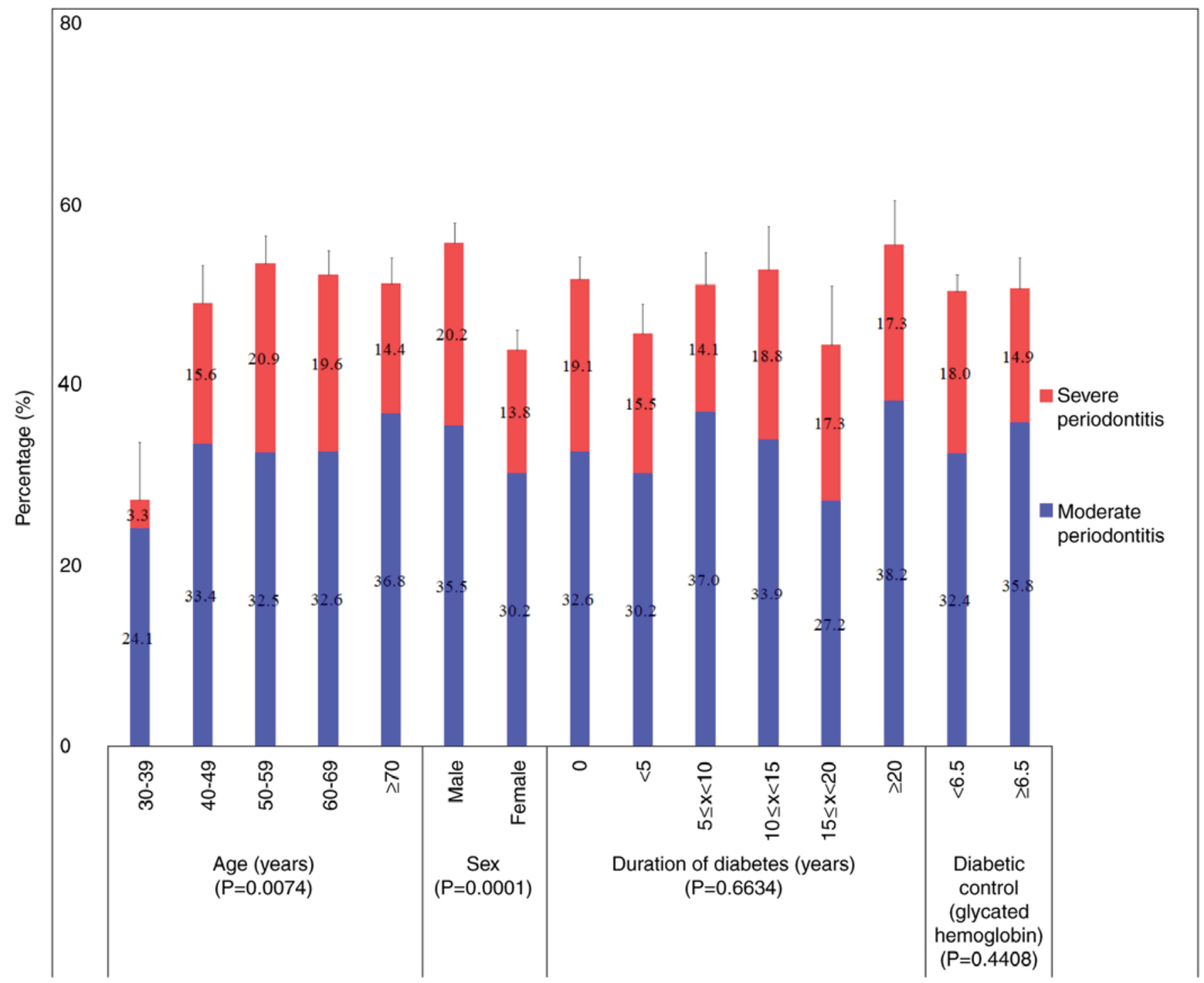

Figure 3. The percentage of the total population of moderate and severe periodontitis categorized by age, sex, duration of diabetes and glycated hemoglobin.

A bidirectional association has been reported between diabetes and periodontitis (3). Patients with diabetes have a higher predisposition to periodontitis (9) and periodontitis is a risk factor in a number of systemic diseases, including diabetes and cardiovascular pathologies (2). In diabetic patients, the elimination of periodontal infections and a reduction in periodontal inflammation produces a noticeable short-term reduction in glycated hemoglobin (32). Individuals with periodontitis had an increased rate of microvascular complications ketoacidosis and of hospitalizations associated with hyperglycemia with odds ratio and $95 \%$ confidence intervals of [2.43 (1.74-3.40)], [2.72 (1.53-4.80)] and $[2.76(1.72-4.42)]$, respectively (33). Treatment of periodontitis reduces the risk of cardiovascular disease in diabetic patients (34). Therefore, periodontitis may stimulate inflammatory changes in adipose tissue and this may create a self-generating cycle of morbidity that links diabetes and periodontal disease (35).

Dental management has a beneficial effect on the health of patients with diabetes (34). Maintaining oral health helps prevent oral chronic diseases and ameliorate the consequences of chronic inflammatory processes (36). Patients with diabetes are likely to visit a physician more frequently than they visit a dentist and it is the physician's responsibility to educate and motivate the patient to seek dental treatment (37).

In conclusion, the results of the present study indicate that age, sex and oral health behavior are risk indicators of periodontitis in Korean adults with diabetes. The present study suggests that that an increased age, being male and engaging in poor oral health behavior increases the risk of periodontitis in participants with diabetes. Further prospective studies involving a larger sample size of diabetic subjects over a longer period of time are required to evaluate the cause-and-effect association. The present study suggests that individuals of a certain age with diabetes should be recommended for regular periodontal evaluation, particularly males.

\section{Acknowledgements}

The authors thank the Korea Centers for Disease Control and Prevention for providing the data. 


\section{Funding}

The present study was supported by Research Fund of Seoul St. Mary's Hospital, The Catholic University of Korea (2017). This work was partly supported by Basic Science Research Program through the National Research Foundation of Korea funded by the Ministry of Science, ICT \& Future Planning (grant no. NRF-2017R1A1A1A05001307; Daejeon, Republic of Korea).

\section{Availability of data and materials}

All data generated or analyzed during this study are included in the published article.

\section{Authors' contributions}

$\mathrm{KH}$ and JP designed the research, analyzed the data and wrote the manuscript. Both authors reviewed the final manuscript.

\section{Ethics approval and consent to participate}

The KNHANES was approved by the Institutional Review Board of the Korea Center for Disease Control and all participants provided written informed consent. The Institutional Review Board at the Catholic University of Korea approved the present study (KC14EISI0636).

\section{Consent for publication}

Not applicable.

\section{Competing interests}

The authors confirm that they have no competing interests.

\section{References}

1. Rajhans NS, Kohad RM, Chaudhari VG and Mhaske NH: A clinical study of the relationship between diabetes mellitus and periodontal disease. J Indian Soc Periodontol 15: 388-392, 2011.

2. Napora M, Grabowska E and Górska R: Prospective analysis of the relationship between the state of periodontal tissues and changes in selected cardiovascular parameters in patients with type 2 diabetes. Adv Clin Exp Med 25: 879-886, 2016.

3. Chapple IL and Genco R; working group 2 of the joint EFP/AAP workshop: Diabetes and periodontal diseases: Consensus report of the joint EFP/AAP workshop on periodontitis and systemic diseases. J Periodontol 84 (4 Suppl): S106-S112, 2013.

4. Khanuja PK, Narula SC, Rajput R, Sharma RK and Tewari S: Association of periodontal disease with glycemic control in patients with type 2 diabetes in Indian population. Front Med 11: 110-119, 2017.

5. Saini R, Saini S and Sugandha R: Periodontal disease: The sixth complication of diabetes. J Family Community Med 18: 31, 2011.

6. Preshaw PM and Bissett SM: Periodontitis: Oral complication of diabetes. Endocrinol Metab Clin North Am 42: 849-867, 2013.

7. Eke PI, Dye BA, Wei L, Slade GD, Thornton-Evans GO, Borgnakke WS, Taylor GW, Page RC, Beck JD and Genco RJ: Update on prevalence of periodontitis in adults in the United States: NHANES 2009 to 2012. J Periodontol 86: 611-622, 2015.

8. Lakschevitz F, Aboodi G, Tenenbaum H and Glogauer M: Diabetes and periodontal diseases: Interplay and links. Curr Diabetes Rev 7: 433-439, 2011.

9. Mesia R, Gholami F, Huang H, Clare-Salzler M, Aukhil I, Wallet SM and Shaddox LM: Systemic inflammatory responses in patients with type 2 diabetes with chronic periodontitis. BMJ Open Diabetes Res Care 4: e000260, 2016.
10. Shetty A, Bhandary R, Thomas B and Ramesh A: A comparative evaluation of serum magnesium in diabetes mellitus type 2 patients with and without periodontitis-a clinico-biochemical study. J Clin Diagn Res 10: ZC59-ZC61, 2016.

11. Nam GE, Kim DH, Cho KH, Park YG, Han KD, Choi YS, Kim SM, Ko BJ, Kim YH and Lee KS: Estimate of a predictive cut-off value for serum 25-hydroxyvitamin D reflecting abdominal obesity in Korean adolescents. Nutr Res 32: 395-402, 2012.

12. Chapter 1: Definition and classification of CKD. Kidney Int Suppl (2011) 3: 19-62, 2013.

13. Kang MS, Kim CH, Jeong SJ and Park TS: Dietary sodium intake in people with diabetes in Korea: The Korean national health and nutrition examination survey for 2008 to 2010. Diabetes Metab J 40: 290-296, 2016.

14. Jeon JY, Ko SH, Kwon HS, Kim NH, Kim JH, Kim CS, Song KH, Won JC, Lim S, Choi SH, et al: Prevalence of diabetes and prediabetes according to fasting plasma glucose and HbAlc. Diabetes Metab J 37: 349-357, 2013.

15. Kingman A, Susin C and Albandar JM: Effect of partial recording protocols on severity estimates of periodontal disease. J Clin Periodontol 35: 659-667, 2008.

16. Park JB, Han K, Park YG and Ko Y: Association between alcohol consumption and periodontal disease: The 2008 to 2010 Korea national health and nutrition examination survey. J Periodontol 85: 1521-1528, 2014.

17. Blinkhorn AS, Choi CL and Paget HE: An investigation into the use of the FDI tooth notation system by dental schools in the UK. Eur J Dent Educ 2: 39-41, 1998.

18. Islam SA, Seo M, Lee YS and Moon SS: Association of periodontitis with insulin resistance, $\beta$-cell function, and impaired fasting glucose before onset of diabetes. Endocr J 62: 981-989, 2015.

19. Albrecht M, Bánóczy J and Tamás G Jr: Dental and oral symptoms of diabetes mellitus. Community Dent Oral Epidemiol 16: 378-380, 1988

20. Bacić M, Plancak D and Granić M: CPITN assessment of periodontal disease in diabetic patients. J Periodontol 59: 816-822, 1988.

21. Emrich LJ, Shlossman M and Genco RJ: Periodontal disease in non-insulin-dependent diabetes mellitus. J Periodontol 62: 123-131, 1991.

22. Schulze A and Busse M: Gender differences in periodontal status and oral hygiene of non-diabetic and type 2 diabetic patients. Open Dent J 10: 287-297, 2016.

23. Kongstad J, Enevold C, Christensen LB, Fiehn NE and Holmstrup P: Impact of periodontitis case criteria: A cross-sectional study of lifestyle. J Periodontol 88: 602-609, 2017.

24. Strauss SM and Stefanou LB: Interdental cleaning among persons with diabetes: Relationships with individual characteristics. Int J Dent Hyg 12: 127-132, 2014.

25. Ervasti T, Knuuttila M, Pohjamo L and Haukipuro K: Relation between control of diabetes and gingival bleeding. J Periodontol 56: 154-157, 1985.

26. Wahi S, Tripathi A, Wahi S, Mishra VD, Singh AP and Sinha N: Assessment of levels of glycosylated hemoglobin in patients with periodontal pathologies: A comparative study. J Contemp Dent Pract 18: 506-509, 2017

27. Bridges RB, Anderson JW, Saxe SR, Gregory K and Bridges SR: Periodontal status of diabetic and non-diabetic men: Effects of smoking, glycemic control, and socioeconomic factors. J Periodontol 67: 1185-1192, 1996.

28. Rylander H, Ramberg P, Blohme G and Lindhe J: Prevalence of periodontal disease in young diabetics. J Clin Periodontol 14: 38-43, 1987.

29. Cerda J, Vázquez de la Torre C, Malacara JM and Nava LE: Periodontal disease in non-insulin dependent diabetes mellitus (NIDDM). The effect of age and time since diagnosis. J Periodontol 65: 991-995, 1994.

30. Han K and Park JB: Association between oral health behavior and periodontal disease among Korean adults: The Korea national health and nutrition examination survey. Medicine (Baltimore) 96: e6176, 2017.

31. Song IS, Han K, Park YM, Ji S, Jun SH, Ryu JJ and Park JB: Severe periodontitis is associated with insulin resistance in non-abdominal obese adults. J Clin Endocrinol Metab 101: 4251-4259, 2016.

32. Grossi SG, Skrepcinski FB, DeCaro T, Zambon JJ, Cummins D and Genco RJ: Response to periodontal therapy in diabetics and smokers. J Periodontol 67 (10 Suppl): S1094-S1102, 1996. 
33. Oliveira LS, Lira-Junior R, Figueredo CM, Gomes MB and Fischer RG: Self-reported periodontitis and complications in type 1 diabetes patients: A Brazilian nationwide survey. Braz Dent J 27: 599-603, 2016.

34. Peng CH, Yang YS, Chan KC, Kornelius E, Chiou JY and Huang CN: Periodontal treatment and the risks of cardiovascular disease in patients with type 2 diabetes: A retrospective cohort study. Intern Med 56: 1015-1021, 2017.

35. Levine RS: Obesity, diabetes and periodontitis-a triangular relationship? Br Dent J 215: 35-39, 2013.
36. Lifshitz F, Casavalle PL, Bordoni N, Rodriguez PN and Friedman SM: Oral health in children with obesity or diabetes mellitus. Pediatr Endocrinol Rev 14: 159-167, 2016.

37. Ummadisetty T, Chava VK and Bhumanapalli VR: Diabetes and periodontitis: How well are the patients aware about an established relation? J Indian Soc Periodontol 20: 472-475, 2016.

(i) (9) This work is licensed under a Creative Commons cc) ${ }_{\mathrm{EY}}$ NC ND Attribution-NonCommercial-NoDerivatives 4.0 International (CC BY-NC-ND 4.0) License. 